\title{
IP-Based Convergence of Fixed and Cellular Networks and Services in the Light of Liberalization
}

\author{
Vergados D., Vayias E., Soldatos J., Drakoulis D. and Mitrou N. \\ Telecommunications Laboratory \\ Department of Electrical Engineering \& Computer Science \\ National Technical University of Athens \\ Heroon Polytechniou 9, Zographou Athens \\ GR 157 73, Greece \\ Tel: +301772 2558 \\ Fax: +301772 2534 \\ Email: \{vergados; evayias\}@telecom.ntua.gr
}

\begin{abstract}
The evolution of an "open" communications universe is now expanding to deliver new communications services, characterised by advanced and ubiquitous, multimedia capabilities. The move by telecommunication companies' corporate customers into the IP world and the need for interoperability between private and public networks will drive service providers and telecom operators to adopt IP in their core networks as a means of unifying traffic types. The particular case addressed in this paper considers a complex yet realistic scenario: the convergence of Fixed/Mobile Communications Provider and Internet Service Provider through the transition to IP technology.
\end{abstract}

Keywords: IP Network, Cellular Operators, Network Services, Convergence, Liberalization of Telecommunications, ISP, ITSP, IP Telephony, VoIP, IP Services

\section{Introduction}

The Internet has emerged during the 1990s as the primary force driving the expansion of demand for telecommunications companies. The growth in Internet usage has led to widespread take-up of Internet Protocol (IP) in corporate LANs, and a trend is now emerging towards IP implementation in corporate WANs as well. The move by telecommunication companies' corporate customers into the IP world, and the need for interoperability between private and public networks, will most propably drive the telecommunication companies to adopt IP in their core networks as a means of unifying traffic types [1]. This trend will be reinforced by emerging and incumbent telecommunication operators short-term need to respond to competition from low-cost IP telephony providers.

Since the IP protocol has been widely adopted for the unification of the different types of underlying physical networks, providing seamless interworking to the application layers, it is of great importance that telecom/cellular operators base their backbone networks on IP technology. Consequently, facing the liberalization of the 
Telecom market, a strategic movement has to be decided towards IP technology both for access and backbone networks, that will allow the deployment of multi-media services over IP (any set of voice, fax, data) in their networks.

Our contribution will initially approach the technical standards related to IP-based control, signalling and delivery of voice, and will then proceed to attempt a technical comparison. The popular ITU-T H.323 and IETF's SIP standards are the focus of this comparison, mainly since the authors believe they are the most interesting pair especially from an academic point of view. A brief reference to QoS for IP networks and RSVP in specific follows. Finally three different scenarios are illustrated - and presented in equal sections, covering the known possible ways of establishing a call by using the current technology: PSTN/mobile terminal to PSTN/mobile terminal; PSTN/mobile terminal to IP Telephony terminal; IP Telephony Terminal to PSTN/mobile terminal.

\section{Technical Approach}

\subsection{Introduction to Technical Approach}

Today's Internet is increasingly used not only for e-mail, ftp and WWW, but also for interactive audio and video services. Historically, voice applications and data applications have required separate networks using different technologies — circuit switching for voice and packet switching for data. In the past decade, much effort has gone into finding a solution that provides satisfactory support for both transmission types on a single network. As a result there has been significant evolution of packetizing technologies for the transmission of voice traffic over data networks.

\subsection{Protocols for Voice / Multimedia Provision over IP Networks}

\section{ITU-T H.323}

The dominant standard for professional VoIP applications, H.323 defines in detail all the components needed for a complying Voice-over-any-packet-network implementation, and has the important advantage of currently being considered a defacto market standard. In contradiction to popular belief H.323 does not define only voice but multimedia provision in general and implementations may be ported with moderate difficulty from one packet-based network to another. Packet-based networks defined by $\mathrm{H} .323$ include IP-based (including the Internet) or Internet packet exchange (IPX)-based local-area networks (LANs), enterprise networks (ENs), metropolitan-area networks (MANs), and wide-area networks (WANs). H.323 can be applied in a variety of mechanisms - audio only (IP telephony); audio and video (videotelephony); audio and data; and audio, video and data. The standard can also be applied to multipoint-multimedia communications. H.323 is a standard that specifies the components, protocols and procedures that will enable the provision of multimedia services over packet switched networks [3]. H.323 is part of a family of 
ITU-T recommendations generally referred to as $\mathrm{H} .32 \mathrm{x}$, where multimedia communication services over a variety of networks are described.

The Version 1 of the H.323 recommendation — visual telephone systems and equipment for LANs that provide a non-guaranteed quality of service (QoS) - was specified by the ITU-T Study Group 16, and accepted in October 1996. Multimedia communications in a LAN environment were the main focus of this work. The emergence of voice-over-IP (VoIP) applications and the lack of a standard for voice over IP resulted in several proprietary and thus incompatible products, while additionally interworking between packet based telephony and conventional circuitswitched telephony was never defined in detail. Version 2 of H.323 emerged to accommodate these additional requirements and was formally accepted in January 1998. The standard shall evolve further and according to analysts new features should cope with performance (faster connections establishment), interworking between different media gateways and other services like fax-over-packet.

\section{Other H.323 associated definitions}

Under H.323 several other recommendations are mentioned here [8] related to

- Audio coding. H.323 defines the support of at least one G.711 codec (audio coding at $64 \mathrm{kbps}$ quality). Additional audio CODEC recommendations such as G.722 (64, 56, and $48 \mathrm{kbps})$, G.723.1 (5.3 and $6.3 \mathrm{kbps})$, G.728 (16 kbps), and G.729 (8 kbps) may also be supported.

- Video coding. H.323 specifies support of video as optional, yet, any H.323 terminal providing video communications must support video encoding and decoding as specified in the ITU-T H.261 recommendation.

- H.225 registration, admission, and status (RAS): Registration, admission, and status (RAS) is the protocol managing connection between any combination of endpoints (terminals and gateways) and gatekeepers. The RAS is used to perform registration, admission control, bandwidth changes, status, and disengage procedures using specific RAS (non-traffic) channels. This signaling channel is opened between an endpoint and a gatekeeper prior to the establishment of any other channels.

- H.225 call signaling: The H.225 call signaling is used to establish a connection between two H.323 endpoints. This is achieved by exchanging H.225 protocol messages on the call-signaling channel. The call-signaling channel is opened between two H.323 endpoints or between an endpoint and the gatekeeper.

- H.245 control signaling: H.245 control signaling is used to exchange end-to-end control messages governing the operation of the H.323 endpoint. These control messages carry information related to the following:

- capabilities exchange

- opening and closing of logical channels used to carry media streams

- flow-control messages

- general commands and indications

- Real-time transfer protocol (RTP): Real-time transport protocol (RTP) provides end-to-end delivery services of real-time audio and video. Whereas H.323 is used to transport data over IP-based networks, RTP is typically used to transport data solely via the user datagram protocol (UDP). RTP, together with UDP, provides 
transport-protocol functionality. RTP provides payload-type identification, sequence numbering, time stamping, and delivery monitoring. UDP provides multiplexing and checksum services.

- Real-time control protocol (RTCP): Real-time transport control protocol (RTCP) is the counterpart of RTP that provides control services. The primary function of RTCP is to provide feedback on the quality of the data distribution. Other RTCP functions include carrying a transport-level identifier for an RTP source, called a canonical name, which is used by receivers to synchronize audio and video.

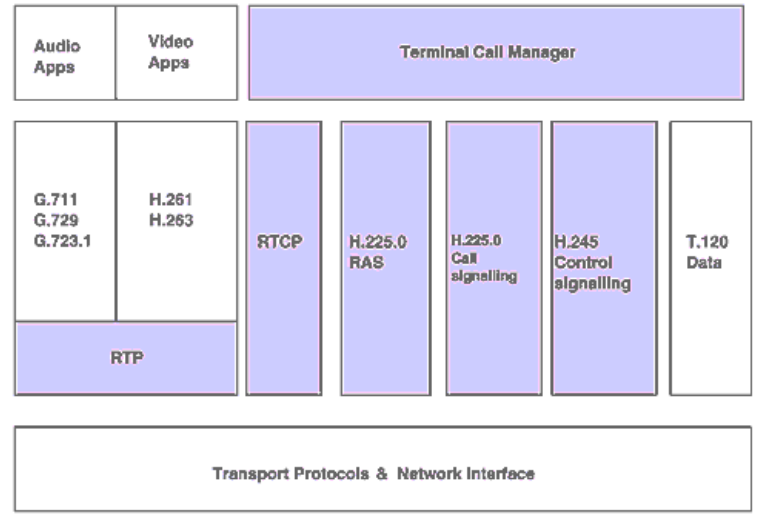

Fig. 1: H.323 protocol stack

\section{The Session Initiation Protocol (SIP)}

The Session Initiation Protocol [4], [7], runs on top of either UDP or TCP, providing its own reliability mechanisms when used with UDP. For addressing, SIP makes use of uniform resource identifiers (URIs), which are generalizations of Uniform Resource Locators (URL's), in common usage in the web. SIP defines its own URI, but its header fields can carry other URIs, such as http, mailto, or phone. SIP is a client-server protocol, similar in both syntax and structure to the well-known HyperText Transfer Protocol (HTTP). However, it defines its own methods and headers for providing the functions required in IP telephony signaling. Requests are generated by one entity (the client), and sent to a receiving entity (the server) that processes them, and then sends responses. This request - response sequence is called a transaction. The end system acting on behalf of a user in SIP is called the user agent. The client portion is called the User Agent Client (UAC) while the server portion is called User Agent Server (UAS). The UAC is used to initiate a SIP request while the UAS is used to receive requests and return responses on behalf of the user. A single host may well act as client and server for the same request. As in HTTP, the client requests invoke methods on the server. Requests and responses are textual, and contain header fields, which convey call properties and service information. SIP reuses many of the header fields used in HTTP, such as the entity headers (e.g., Content-type) and authentication headers.

Three types of servers may be found within a SIP network: Registration, Proxy and Redirect Servers. A registration server receives updates concerning the current locations of users. A proxy server upon receiving a request forwards it to the next-hop 
server, which has more information about the location of the called party. Thus the proxy servers may be held responsible for call routing. A redirect server on receiving request, determines the next-hop server and returns the address of the next-hop server to the client instead of forwarding the request. We will review the technical essence of SIP in a following section, by comparing it to the H.323.

\subsection{Network Elements - Components defined by ITU}

The H.323 standard specifies four kinds of network components, which provide the point-to-point and point-to-multipoint multimedia-communication services:

- Terminals: Used for real-time bi-directional multimedia communications, an H.323 terminal may come in many forms, e.g. as a PC application or a standalone device, running an H.323 and the multimedia applications. The terminal should basically support audio communications and optionally video or data communications. The primary goal of H.323 is to allow the required interworking features with other multimedia terminals. In the ITU standards world, H.323 terminals are compatible with H.324 terminals on SCN and wireless networks, H.310 terminals on B-ISDN, H.320 terminals on ISDN, H.321 terminals on BISDN, and H.322 terminals on guaranteed QoS LANs. H.323 terminals may be used in multi-point conferences.

- IP Telephones: An IP Telephone is a telephone device that is able to transports voice over an IP network using data packets instead of circuit switched connections over voice only networks. An IP Telephone consists of the following components: User Interface, Voice Interface, Network Interface (IP), and Processor Core and associated logic.

- Gateways: One of the primary issues the developers of the H.323 standard had to deal with, was interoperability with other multimedia-services networks. This interoperability is achieved through the use of a gateway. A gateway thus connects two dissimilar networks. This connectivity of dissimilar networks is achieved by translating protocols for call setup and release, converting media formats between different networks, and transferring information between the networks connected by the gateway. A gateway is not required, however, for communication between two terminals on an H.323 network. Also, internet telephony gateways consist of two functional parts - a dumb media gateway which converts audio data and an intelligent media gateway controller which communicates with the rest of the world over signaling protocols and controls 1$\mathrm{N}$ media gateways over a gateway control protocol. ITU-T has already standardized a Gateway Control Protocol called as H.GCP protocol, which is used to control Media Gateways.

- Gatekeepers: A gatekeeper is a central, coordinating point within the H.323 network. Although they are not required by the standard, and are not included in some gatekeepers provide important services such as addressing, authorization and authentication of terminals and gateways; bandwidth management; accounting; billing; and charging. Gatekeepers may also provide call-routing services. 
- Multipoint control units (MCUs): MCUs provide support for conferences of three or more H.323 terminals. All terminals participating in the conference establish a connection with the MCU. The MCU manages conference resources, negotiates between terminals for the purpose of determining the audio or video coder/decoder (CODEC) to use, and may handle the media stream.

It should be emphasized at this point that although gatekeepers, gateways, and MCUs are logically separate components of the H.323 standard, they may be implemented as a single physical device.

\section{Ease of Implementation, Compatibility and Scalability}

Having briefly presented the major existing standards for VoIP, we will proceed by attempting a comparison on issues like ease of implementation, compatibility and scalability, and by referring to the engineering approaches in the implementation that result in these differences [5].

\section{IETF SIP vs. ITU-T H.323}

Ease of Implementation: H.323 is a rather complex protocol while SIP, being similar to the HTTP syntax and message exchange, is a lighter, easier to implement protocol. H.323 defines numerous (hundreds!) components while SIP defines different header types with similar structure. SIP messages are encoded as plain text, similar to HTTP. This leads to simple parsing and generation, and development can be based on languages like Perl. H.323 on the other hand uses binary representations for its messages based on ASN.1 (Abstract Syntax Notation) and the packed encoding rules (PER). Furthermore under H.323 several interactions between standard components (e.g. H.225, H.245), internally in the protocol are possible increasing the complexity.

Compatibility: SIP has built in a rich set of extensibility and compatibility functions. Compatibility is still maintained across different versions. As SIP is similar to HTTP, mechanisms being developed for HTTP extensibility can also be used in SIP. Among these is the Protocol Extensions Protocol (PEP), which contains pointers to the documentation for various features within the HTTP messages. SIP uses the Session Description Protocol (SDP) to convey the codecs supported by an endpoint in a session. H.323 provides extensibility mechanisms as well. These are generally nonstandard Param fields placed in various locations in the ASN.1. These params contain a vendor code, followed by an opaque value, which has meaning only for that vendor. This allows for different vendors to develop their own extensions. However extensions are limited only to those places where a non-standard parameter has been added. If a vendor wishes to add a new value to some existing parameter, and there is no placeholder for a nonstandard element, one cannot be added. Secondly, although H.323 requires full backward compatibility from each version to the next it has no mechanisms for allowing terminals to exchange information about which extensions each version supports. In H.323, each codec must be centrally registered and standardized. As many of these carry significant intellectual property, there is no free, 
sub-28.8 kb/s codec which can be used in an H.323 system. This presents a significant barrier to entry for small players and universities.

Scalability: SIP and H.323 differ in terms of scalability. SIP servers and gateways will need to handle many calls. For large, backbone IP telephony providers, the number of calls being handled by a large server can be significant. In SIP, a transaction through several servers and gateways can be either stateful or stateless. In the stateless model, a server receives a call request, performs some operation, forwards the request, and completely forgets about it. SIP messages contain sufficient state to allow for the response to be forwarded correctly. SIP can be carried on either TCP or UDP. In the case of UDP, no connection state is required. This means that large, backbone servers can be based on UDP and operate in a stateless fashion, reducing significantly the memory requirements and improving scalability. SIP is simpler to process than H.323; given the same processing power, SIP should allow more calls per second to be handled on particular box than H.323. As H.323 was originally conceived for use on a single LAN and thus for large numbers of domains, and complex location operations, it is normal to expect the appearance of scalability problems. As a practical example in an H.323 system, both telephony gateways and gatekeepers will be required to handle calls from a multitude of users and furthermore the connections established are TCP based, which means a gatekeeper must retain its TCP connections. H.323 will have a hard time coping with a multi-gateway situation a case where other protocols like the multi-gateway MGCP/MeGaCo shall be deployed.

\section{QOS over IP Networks - The RSVP Protocol}

The IP has become a ubiquitous communications universal network that intends to incorporate traditional data-oriented services together with new multimedia services (IP telephony, videotelephony, multimedia, etc.). Within the framework of this global information infrastructure deployment, the demand for IP connectivity services suitable for business is increasing. Several QoS frameworks have been proposed in an attempt to provide IP services of a quality level suitable for multimedia and businesscritical communication purposes [9],[10]. Some of these proposals have also addressed the issues of seamlessly provisioning and dynamically configuring IP services. A prominent position among these frameworks is held by the Integrated Services Architecture (IntServ) that works in conjunction with the Resource reSerVation Protocol (RSVP) towards providing QoS guarantees in a per flow basis [11]. Another important QoS framework is the Differentiated Services model (DiffServ). DiffServ attempts to provide scalable (i.e. no per-flow signaling or state) service differentiation in the IP Networks. Even though a lot of effort has been allocated to developing these frameworks, it seems that both present some drawbacks, which may hinder their applicability towards providing really quantitative, end-to-end QoS in a scalable manner. Specifically, the IP Network community has voiced concern over the applicability and scalability of RSVP and the Integrated Services model in the global IP Network infrastructure. The need for maintaining state and applying traffic control functions for every single connection and at every network 
element is considered as an obstacle to the IP Network applicability of the method. On the other hand, although the DiffServ architecture presents a great potential towards achieving scalable service provision in the next generation networks, it still lacks specific traffic control and resource management strategies for guarantying quantitative, end-to-end QoS metrics. How to control the traffic in order to achieve the advertised per-hop behavior is still not clearly defined in the DiffServ environment. Thus, the IP Networks are still restricted to offering the traditional best effort data forwarding service, which cannot meet the emerging QoS demands.

\section{The RSVP Protocol}

The network delay and Quality of Service are the most hindering factors in the voicedata convergence. The most promising solution to this problem has been developed by IETF viz., RSVP. RSVP allows prioritization and latency guaranties to specific IP traffic streams. RSVP enables a packet-switched network to emulate a more deterministic circuit switched voice network. With the advent of RSVP, VOIP has become a reality today. RSVP requests will generally result in resources being reserved in each node along the data path. RSVP requests resources in only one direction, therefore it treats a sender as logically distinct from a receiver, although the same application process may act as both a sender and a receiver at the same time. RSVP is not itself a routing protocol, it is designed to operate with current and future unicast and multicast routing protocols. RSVP interacts with entities called the packet classifier and the packet scheduler installed on the host to make quality of service decisions about the packets sent in by applications. It first queries the local decision modules to find out whether the desired QoS can be provided (this may involve resource-based decisions as well as policy-based decisions). It then sets up the required parameters in the packet classifier and the packet scheduler. The packet classifier determines the route of the packet, and the scheduler makes the forwarding decisions to achieve the desired QoS. In case the link layer at the host has its own QoS management capability, then the packet scheduler negotiates with it to obtain the QoS requested by RSVP.

The central component of the RSVP architecture, which applications interact with, is a flow specification, or flowspec, which describes both the traffic stream sent out by the source as well as the service requirements of the application. These flowspecs actually specify the desired QoS, and are used to set the parameters in the node's packet scheduler. In addition to these there are filter specs which together with the session specification define (or rather filter) the data packets which are to receive the QoS defined by the flowspecs. These are basically a set of filters, which can be used to select arbitrary subsets of packets in a given session, by selecting any fields in protocol headers, application headers etc. Data packets that are addressed to a particular session but which do not match any of the filter specs for that session are handled as best-effort traffic.

If IP Telephony were to move into the mainstream, the technology would have to deliver predictable, consistent voice quality over any network infrastructure. This meant successfully addressing a myriad of issues. Most of them are still under extensive study. The main issue is to provide QoS in every call establishment. Major standards organizations and private consortiums are putting much effort in this direction and their results should be monitored throughout the paper time period. 


\section{The Proposed Network Architecture}

\section{The Key Issues}

This article addresses a complex scenario involving Telecom Operators of fixed network, or alternatively, Cellular Operators and Internet Service Providers. In particular, it describes in detail different types of services that should be offered by the operators and proposes various interworking schemes between the ISP network and the fixed/cellular conventional (circuit switched) network, in an IP-integrated network environment. The interworking scenario focuses both in technical aspects, and on related marketing implications based on analysis by industry experts and market analysts. In particular, opinions expressed within the paper are perfectly aligned to the following principles:

- The market for long distance services based on IP infrastructure is about to explode

- ISPs must consider the need to upgrade their infrastructure in order to turn to Internet Telephony Service Providers (ITSPs) by offering IP telephony

- By unfying voice, fax and corporate data traffic, IP-based network operators face significant profit opportunities and become a significant competitor to circuit switched telecommunication companies

ISPs may use a single infrastructure for providing both, Internet access and IP Telephony. Data-oriented switches shall be deployed for switching both data and packetized voice. IP is more fit and cost-effective for the delivery of voice services than other techniques like e.g. ATM, since ATM reserves bandwidth for Constant Bit Rate (CBR) applications like voice circuits, even when this circuit is not used [2].

\section{The Key Areas of Application}

Most of the focus on VoIP is currently centered on two key applications.

The first is private business network applications. Businesses with remotely located branch offices, which are already connected together via a corporate intranet for data services can take advantage of the existing intranet by adding voice and fax services using VoIP technologies. Businesses are driving the demand for VoIP solutions primarily because of the incredible cost savings that can be realized by reducing the operating costs of managing one network for both voice and data and by avoiding access charges and settlement fees, which are particularly expensive for corporations with multi-international sites. Managed corporate intranets do not have the QoS issues, which currently plague the Internet; thus voice quality approaches toll quality.

The second key application is VoIP over public networks. This application involves the use of voice gateway devices designed to carry voice to Internet Service Providers, who are regularly referred to as Internet Telephony Service Providers, or to the emerging Next Generation Carriers, developing IP networks specifically to carry 
multimedia traffic such as VoIP. ISPs are interested in VoIP as a way of offering new value-added services to increase their revenue stream and break out of the low monthly fixed fee structure currently in place for data services (a realistic example is depicted in Figure 2).

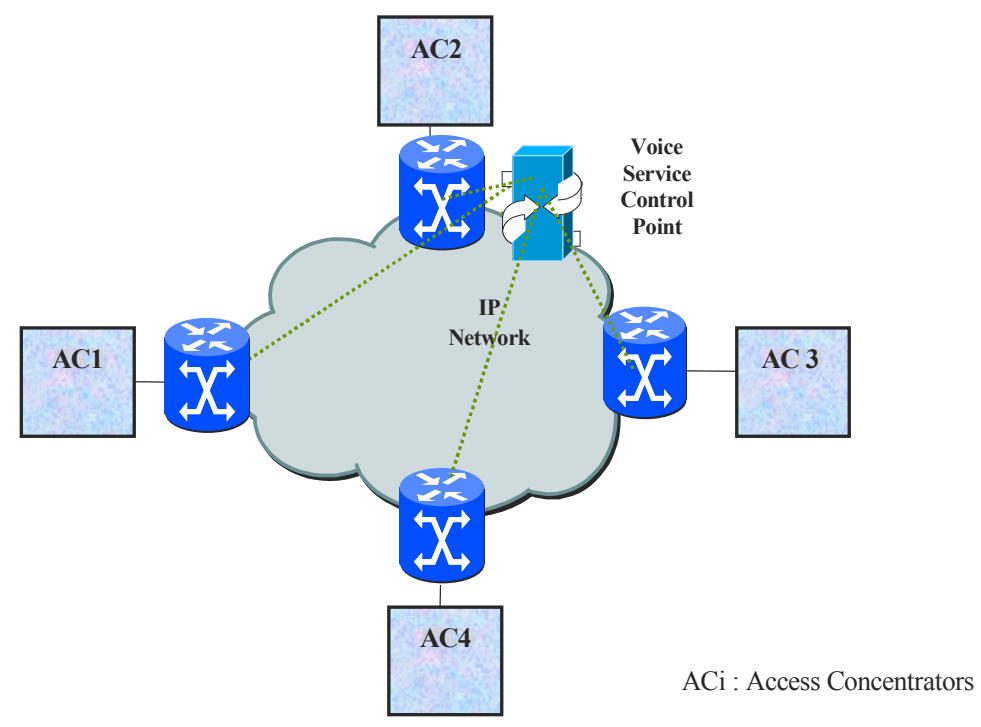

Fig. 2: The Converged Network

VoIP also allows the improvement of network utilization. These new services include voice and fax on a per-minute usage basis at rates significantly less than voice and fax rates for service through the PSTN. The sustainability of this price advantage may be short term, and is dependent on whether the regulatory agencies will require ISPs to pay the same access charges and settlement fees, PSTN carriers are obligated to pay. New carriers are interested in VoIP because data networks are more efficient than traditional voice networks. In the near term, these new carriers can avoid the access charges and settlement fees, which account a sufficient part of the cost of a long distance call. IP Telephony (IPT) networks that offer competitive voice and fax services have been the predominant new business opportunity over the last years, as vendors have introduced the equipment and functionality necessary to deploy large scale services. Now, customers may take advantage of flat Internet rating vs. hierarchical PSTN rating and save money while letting their long-distance calls be routed over Internet. The IPT users may also profit of its applications'-oriented nature: software solutions may be easily extended and integrated with other services and applications, e.g. whiteboarding, electronic calendar, or browsing. 


\section{Interoperability Scenarios}

Technology in our days is mature enough to develop the right software and hardware components for supporting interoperability among different networks. The following figure (Fig. 3) illustrates the physical components with regard to the H.323 standard.

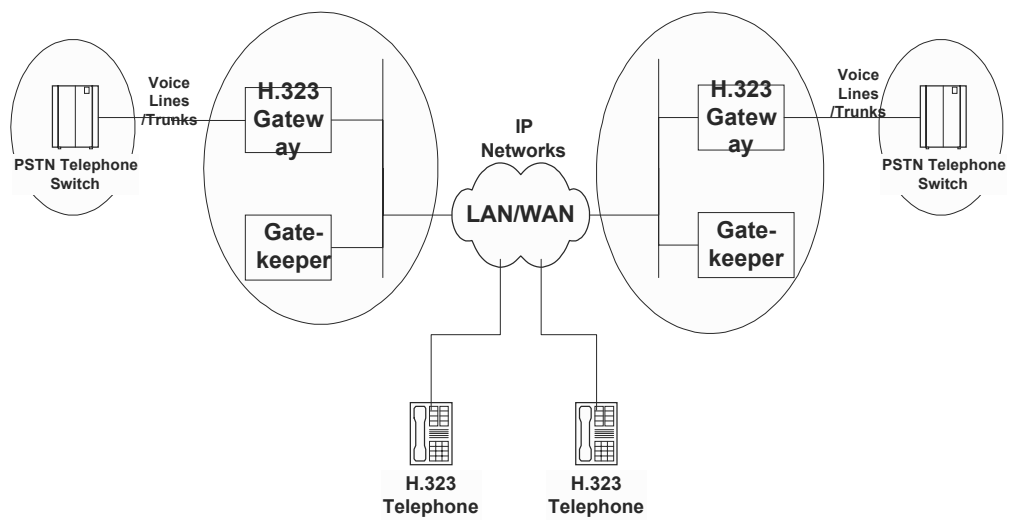

Fig. 3: A basic ITS infrastructure block diagram

As a practical example of the applicability of the presented concepts, three different scenarios of interoperability will be illustrated, covering the known possible ways of establishing a call by using the current technology. These are the following, which are going to be described in sections that follow:

- PSTN/mobile telephone to PSTN/mobile telephone

- PSTN/mobile telephone to IP Telephony terminal

- IP Telephony terminal to PSTN/mobile terminal

It is important to note before examining the scenarios that as it should be obvious by the above figure, the structure of any solution used for delivering voice traffic is only different at the end-points of the H.323 gateways. At these points only, signalling traffic from both sides is 'terminated' and specific medium-dependent hardware is installed.

\section{PSTN/Mobile Telephone to PSTN/Mobile Telephone}

1. An Internet Telephony Service subscriber is able to dial an access number provided by the Internet Telephony Service Provider.

2. The call is routed by the PSTN/Mobile to the "access" Internet telephony switch.

3. The gateway plays an announcement requesting that the subscriber enter the destination telephone number to be called.

4. The destination digit information is sent to the gatekeeper.

5. The gatekeeper determines a destination gatekeeper IP address based on the destination digit information. 
6. An IP packet requesting the availability status of the destination gateway is sent to the destination gatekeeper.

7. The destination gatekeeper responds to the request by providing destination gateway availability and IP address information.

8. The originating gatekeeper then transfers this information to the originating gateway.

9. The originating gateway sets up a virtual circuit to the destination gateway.

10. This circuit is identified by a call reference variable (CRV) that will be used by both gateways for the duration of the call to identify all IP packets associated with this particular call.

11.The destination gateway selects an outgoing PSTN/Mobile voice trunk and signals to the PSTN/mobile switch to attempt to setup a call to the dialed telephone number.

12.If the PSTN/Mobile switch signals that the call setup is successful and the called party has answered, IP signaling messages are relayed to the originating gatekeeper.

13.The gatekeeper then signals the originating gateway, which in turn signals the originating PSTN/Mobile switch to indicate that the call is now completed.

In the IP network, voice packets are exchanged between the gateways for the duration of the call.

\section{PSTN/Mobile Telephone to IP Telephone}

Next we browse through a sample call flow, that originates from the PSTN/Mobile and terminates at an IP-based H.323 terminal.

1. An Internet telephony subscriber dials an access number that has been provided by the Internet telephony service provider.

2. The call is routed by the PSTN/Mobile to the "access" Internet telephony switch.

3. The gateway plays an announcement requesting that the subscriber enter the destination number to be called. (In this case, the telephone number is assigned to an H.323 terminal.)

4. The collected destination digit information is sent in a call setup request message to the gatekeeper.

5. The gatekeeper determines a destination gatekeeper IP address based on the destination digit information.

6. An IP packet requesting the availability status for the destination H.323 terminal is sent to the destination gatekeeper.

7. The destination gatekeeper responds to the request by providing the destination terminal availability status and IP address information to the originating gateway.

8. The originating gateway sets up a virtual circuit to the destination H.323 terminal. This circuit is identified by a call-reference variable that will be used by both the originating gateway and the H.323 terminal for the duration of the call to identify all IP packets associated with this particular call.

9. If the H.323 terminal indicates that call setup is successful and the called party has answered, IP signaling messages are sent to the originating gatekeeper, which then signals the originating gateway.

10.The originating gateway signals the originating PSTN/Mobile switch to indicate that the call is now completed. 
The exchange of IP packets proceeds until either the calling or called party terminates the call.

\section{IP Telephony Terminal to PSTN/Mobile Telephone}

Following let us trace a sample call flow, which originates from an IP-based H.323 terminal (e.g., a personal computer) and terminates in the PSTN/Mobile.

1. An Internet telephony subscriber initiates an Internet telephony call setup procedure at an H.323 terminal (e.g., a PC).

2. The H.323 terminal sends an IP packet specifying the destination digits information to a preselected gatekeeper.

The call scenario from this point onward is similar to steps four through eight described in the PSTN/mobile to IP to PSTN/Mobile call flow (replace "originating gateway" with "originating terminal").

\section{Conclusions - Expected Results}

The main problem that was presented analytically in this paper, is how networks with different communication profiles will be operating under a mature and integrated way. That requires that a number of problems have to be solved. First, communication among the different nodes has to be clarified. The proposed interworking architecture explains that a signaling communication among a number of nodes needs to be established before the path is identified. In addition, there are further responsibilities that have to be affiliated, such as authentication, authorization, Quality of Service, tariffing and billing. In our proposed network, the customer management and billing system for IP telephony needs to be able to support reliable authentication mechanisms to identify the user uniquely, and be able to modify those mechanisms in real time. Also, in the converged IP telephony, the service provider may wish to check a variety of criteria before provisioning the service to the user. Customer management and billing systems used should allow the ITSP to perform real-time authorization before the service is made available to the user. The market will soon require the ability to choose their "quality of service", paying more for higher quality and reliability, or less for lower quality needs such as personal calls or one-way communication (speaker delivering a presentation). The customer management and billing system should able to support the ITSP's need to route delay-sensitive voice and video traffic. As a result, monitoring and controlling interconnection charges is key for tariffing and billing mechanisms. To maximize customer benefits, interconnection rules should be independent of technology and network topology. Authorities must ensure rules applied to the conventional Public Switched Telephone Network (PSTN) are also applied to fixed/mobile networks and IP Networks.

Consequently, the creation of the new telephony services in the proposed converged network will be must faster, cheaper and easier. In particular, an extensive technical analysis of the proposed network was presented, the open issues are identified and the problems arising are discussed in detail and proper solutions are proposed. The results of this paper together with the strategic movement to IP technology both for access and backbone networks, are of a great importance for the evolution of a new, "open" 
communications universe, which is now coming together harmoniously to deliver new communications services, characterized by mobility and advanced multimedia capabilities.

\section{References}

1. H. Schulzrinne and J. Rosenberg, "Internet telephony: Architecture and protocols -- An IETF perspective", Comp. Networks and ISDN Sys., vol. 31, Feb. 1999, pp. 237-55.

2. J. Estrin and S. Casner," Multimedia Over IP: Specs Show the Way", Data Communications, Aug. 1996.

3. ITU-T Rec. H.323, "Visual telephone systems and equipment for local area networks which provide a non-guaranteed quality of service," Geneva, Switzerland, May 1996.

4. M. Handley et al., "SIP: session initiation protocol," IETF 2543, Mar. 1999.

5. H. Schulzrinne and J. Rosenberg, "A comparison of SIP and H.323 for internet telephony," Proc. NOSSDAV, Cambridge U.K., July 1998.

6. H. Schulzrinne et al., "RTP: a transport protocol for real-time applications", IETF RFC 1889, Jan. 1996.

7. H. Schulzrinne and J. Rosenberg, "The session initiation protocol: Providing advanced telephony services across the internet," Bell Labs Tech. J., vol. 3, Oct.-Dec. 1998, pp. $144-60$.

8. J. Toga and J. Ott, "ITU-T standardization activities for interactive multimedia communications on packet networks: H.323 and related recommendations," Comp. Networks, vol. 31, no. 3, 1999, pp. 205-23.

9. Busse, B. Deffner, and H. Schulzrinne, "Dynamic QoS control of multimedia applications based on RTP", Comp. Commun., vol. 19, Jan. 1996, pp. 49-58.

10. Shenker, S., C. Partridge and R. Guerin, "Specification of Guaranteed Quality of Service", RFC 2212, September 1997.

11. Wroclawski, J., "The Use of RSVP with IETF Integrated Services", RFC 2210, September 1997.

12. B. Douskalis, IP Telephony, HP Professional Books, Prentice Hall, 2000. 